\title{
Ultrafast Excitation of an Inner-Shell Electron by Laser-Induced Electron Recollision
}

\author{
Yunpei Deng, ${ }^{1}$ Zhinan Zeng, ${ }^{2,3}$ Zhengmao Jia, ${ }^{2}$ Pavel Komm, ${ }^{4}$ Yinhui Zheng, ${ }^{2}$ Xiaochun Ge, ${ }^{2}$ \\ Ruxin $\mathrm{Li}^{2,3,5, *}$ and Gilad Marcus ${ }^{4, \dagger}$ \\ ${ }^{1}$ SwissFEL, Paul Scherrer Institut, 5232 Villigen PSI, Switzerland \\ ${ }^{2}$ State Key Laboratory of High Field Laser Physics, Shanghai Institute of Optics and Fine Mechanics, \\ Chinese Academy of Sciences, Shanghai 201800, China \\ ${ }^{3}$ IFSA Collaborative Innovation Center, Shanghai Jiao Tong University, Shanghai 200240, China \\ ${ }^{4}$ Department of Applied Physics, Benin School of Engineering and Computer Science, \\ Hebrew University of Jerusalem, Jerusalem 91904, Israel \\ ${ }^{5}$ School of Physical Science and Technology, ShanghaiTech University, Shanghai 200031, China
}

(Received 13 October 2015; published 18 February 2016)

\begin{abstract}
Extreme ultraviolet attosecond pulses, generated by a process known as laser-induced electron recollision, are a key ingredient for attosecond metrology, providing a tool to precisely initiate and probe subfemtosecond dynamics in atoms, molecules, and solids. However, extending attosecond metrology to scrutinize the dynamics of the inner-shell electrons is a challenge, that is because of the lower efficiency in generating the required soft x-ray $(\hbar \omega>300 \mathrm{eV})$ attosecond bursts. A way around this problem is to use the recolliding electron to directly initiate the desired inner-shell process, instead of using the currently low flux x-ray attosecond sources. Such an excitation process occurs in a subfemtosecond time scale, and may provide the necessary "pump" step in a pump-probe experiment. Here we used a few cycle infrared $\left(\lambda_{0} \approx 1800 \mathrm{~nm}\right)$ source and observed direct evidence for inner-shell excitations through the laser-induced electron recollision process. It is the first step toward time-resolved core-hole studies in the $\mathrm{keV}$ energy range with subfemtosecond time resolution.
\end{abstract}

DOI: 10.1103/PhysRevLett.116.073901

Most of light-matter interaction processes, within the limits of "low" frequency and a few-photon interaction, are well described by the single-active-electron approximation. In this approximation the dynamic of only one valence electron is considered, while the effect of the rest is taken as an averaged masking charged cloud. On the other hand, excitations of electrons from deeper shells are usually accompanied by multielectron dynamics such as double excitation, the Auger decay, Cooper minima, and the giant resonance, which cannot be explained by the single active electron approximation. Such excitations are unstable and usually decay on a time scale ranging from few femtoseconds to a few attoseconds $[1,2]$. The decay may take place in a single step, but more often occurs as a cascade of radiative and nonradiative channels. Spectroscopic data may give some general information about the nature of such dynamics but often fail to follow the exact details, for example, the line widths of the cascade Auger decays reveal the total decay rate but not the order of decaying channels and their individual decay rate. To really follow such dynamics, one resorts to a time domain spectroscopy [2-4], in which a first "pulse" initiates the process and a second "pulse" probes it. Since the relevant time scale for such dynamics spans from attoseconds to femtoseconds and the relevant energy scale spans from $10^{2}-10^{5} \mathrm{eV}$, x-ray attosecond bursts may be the choice to serve as the pump and the probe events. However, with the low photon flux of current soft x-ray attosecond sources $(\hbar \omega>300 \mathrm{eV})$ and the low absorption cross sections in this spectral range, it is currently impossible to both pump and probe these processes with attosecond $\mathrm{x}$-ray pulses. To probe processes involving valence electrons, an ultrashort infrared pulse is often used to initiate the process, and a well synchronized extreme ultraviolet (XUV) attosecond pulse probes it [5]. It is difficult to extend this scheme to excite inner-shell processes because of the large energy difference between inner-shell energies and the infrared photon energy. Excitation of inner-shell dynamics by laser-induced electron recollision might be the solution. Here we show direct evidence for such excitations, as opposed to previous indirect evidence $[6,7]$. Such an excitation process occurs in a subfemtosecond time scale, and thus, provides the necessary "pump" step and might become the key for future "pump-probe" studies of inner-shell dynamics.

Insight into the laser-induced electron recollision process is given in a semiclassical model [8,9], in which, the electron is first tunnel ionized by the strong electromagnetic laser field and then it is accelerated forth and back by the same alternating field to return to its parent ion with excess kinetic energy. The outcome of this recollision may split into three different channels (see Fig. 1): the first is recombination with the parent ion while emitting energetic electromagnetic radiation (high order harmonic and XUV attosecond pulse generation); the second is elastic scattering, which manifests itself in a discrete energy-spectrum of 


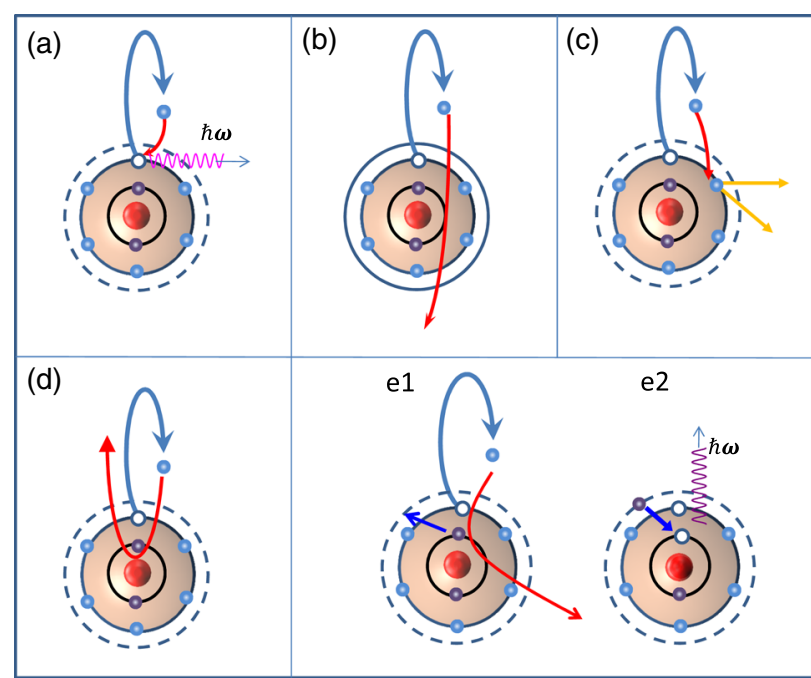

FIG. 1. The different outcomes from the laser-induced electron recollision process. (a) The returning electron recombines with the hole left in the valence shell and release its excess energy as a XUV photon of the high order harmonics. (b) The electron is weakly scattered by the parent ion (elastic scattering) leading to the discrete ATI spectrum. (c) The returning electron kicks out another electron (NSDI). (d) A large-angle scattering of the electron by the parent ion followed by soft $\mathrm{x}$-ray emission due to the strong charge acceleration (bremsstrahlung radiation). (e) The returning electron excites or ionizes an inner shell electron, leaving a hole in this shell (e1). After a while, this excited electron recombines with the core-hole and may emit a soft $\mathrm{x}$-ray photon or decay by another nonradiative process (e2).

the scattered electron, known as above threshold ionization (ATI [10]); and the third channel is an inelastic scattering, that results in additional excitation or ionization. Indeed, only a few years after the discovery of ATI and the high order harmonic $(\mathrm{HOH})$, the accompanying "nonsequential double ionization" (NSDI) process was discovered [11,12]. While both ATI and the $\mathrm{HOH}$ may well be explained in the single-active-electron approximation through the above mentioned three-steps model, NSDI is the first evidence for laser-induced inelastic recollision in which the returning electron kicks out another electron. The same returning electron may be used to initiate ultrafast processes in atoms and molecules with a subfemtosecond time resolution [13]. If the recolliding electron gains enough kinetic energy, it may also initiate inner-shell excitations.

In our experiment we focus a $12 \mathrm{fs}, 1 \mathrm{~mJ}$ infrared radiation source $\left(\lambda_{0}=1.8 \mu \mathrm{m}\right)$ on a pulsed gas jet and observed the soft $\mathrm{x}$-ray radiation from the interaction region. The reason for using an infrared laser instead of $800 \mathrm{~nm}$ laser is the quadratic scaling of the ponderomotive energy with the wavelength of the drive laser. According to the semiclassical three steps model, the maximum energy an electron may come back with, when it collides with the parent ion, is equal to $3.17 U_{p} \propto I_{0} \lambda^{2}$. Here, $U_{p}$ is the ponderomotive energy, $I_{0}$ and $\lambda$ are the peak intensity and the central wavelength of the drive field, respectively.
Because of this quadratic scaling, there is now a great interest in developing and using few-cycle radiation sources having longer wavelengths than $800 \mathrm{~nm}$. Such infrared sources have already demonstrated the extension of $\mathrm{HOH}$ spectra towards the soft $x$-ray range $(\hbar \omega>1 \mathrm{keV})$ [6]. The quadratic dependent of the ponderomotive energy with the drive wavelength also proves to be useful in generating a bright and compact incoherent hard x-ray source, by focusing sub-100 fs infrared pulses on a solid target [14]. The infrared radiation source we have used here is based on an optical parametric amplifier, described in detail elsewhere [15]. The soft $\mathrm{x}$-ray radiation that is coming from the excited atoms is a measure of the amount of excitation, but in order to separate it from the accompanying $\mathrm{HOH}$ radiation, we observed the soft $\mathrm{x}$-ray radiation at a right angle to the infrared propagation direction. The infrared beam was focused on the gas target placed inside a vacuum chamber by a $f=300 \mathrm{~mm} \mathrm{CaF}$ lens to a spot size of about $65 \mu \mathrm{m}$ FWHM, which results in a peak intensity of about $3 \times 10^{15} \mathrm{~W} / \mathrm{cm}^{2}$. The corresponding ponderomotive energy is about $900 \mathrm{eV}$, enough to excite the $K$ shell of neon and the $L$ shell of krypton. For the gas target we used jets of neon and krypton from a pulsed nozzle (series 9 Parker nozzle, orifice diameter of $350 \mu \mathrm{m}$ and backing pressures ranging from 1-10 bar), the pulsed nozzle was operated at $20 \mathrm{~Hz}$ to keep the pressure inside the vacuum chamber below $10^{-3}$ mbar. Soft x-ray spectra from the krypton and the neon atom were recorded by a silicon drift detector (Amptek XR-100SDD) with a $12.5 \mu \mathrm{m}$ beryllium window, positioned $350 \mathrm{~mm}$ away from the interaction region. This kind of detector [16] is working in the photoncounting mode, while at the same time it is able to measure the photon energy. Because of the statistical nature of photon counting, at low counts, the spectra may look noisy. For the sake of clarity we present here smoothed (moving average) spectra, and give the raw spectra in the Supplemental Material [17]. During the experiment, we scanned the focus position along the gas propagation direction to find the best $\mathrm{x}$-ray yield. We obtained the best $\mathrm{x}$-ray yield when we placed the beam focus just next to the nozzle exit; all of the results in this Letter were taken at this position.

Figure 2 shows the soft $\mathrm{x}$-ray spectra coming from $\mathrm{Ne}$ and $\mathrm{Kr}$ targets. The spectrum shows the characteristic $K$-shell line from $\mathrm{Ne}$ and $L$-shell line from $\mathrm{Kr}$, on top of a wider continuum. The continuum radiation from $\mathrm{Kr}$ extends up to $\sim 2800 \mathrm{eV}$ which is in good agreement with the above mentioned formula for the maximum possible kinetic energy of the recolliding electron: $\mathrm{K}_{\max }=$ $3.17 U_{p}=2.96 \times 10^{-13} I_{0} \lambda^{2}$ (K is in $\mathrm{eV}, I$ is in $\mathrm{W} / \mathrm{cm}^{2}$ and $\lambda$ is in $\mu \mathrm{m})$. We speculate that the origin of this continuum is either coming from the recombination radiation or from the bremsstrahlung radiation. The sharp cutoff at the lower energy end is due to absorption in the beryllium window. 


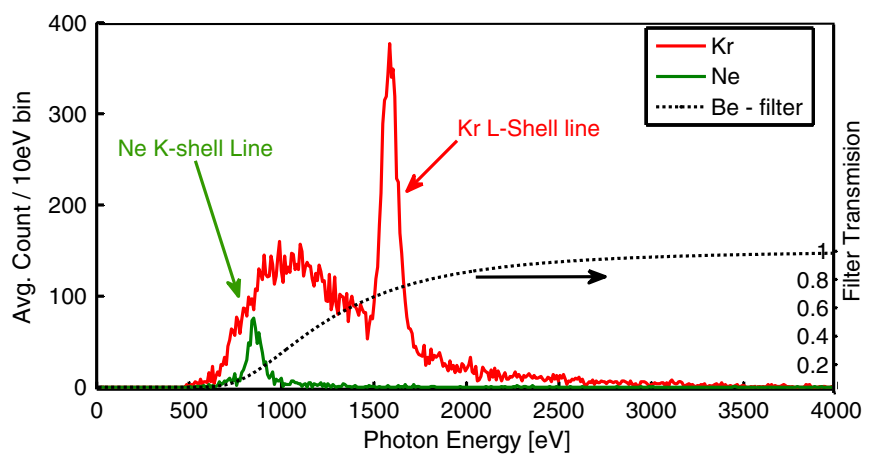

FIG. 2. The fluorescence spectra from the excited Ne (green line) and $\mathrm{Kr}$ (red line) atoms. The signal from the $\mathrm{Ne}$ is mainly from the $K$-shell transition with a weak continuum that stretches up to $\approx 1600 \mathrm{eV}$. In the $\mathrm{Kr}$ spectrum there is a sharp line, belonging to the $L$-shell transition, on top of a pronounced continuum. (The resolution of the SDD detector is not high enough to resolve the $L_{\alpha}, L_{\beta}$ splitting.) The above Ne spectrum and the $\mathrm{Kr}$ spectrum were taken at different conditions. The $\mathrm{Kr}$ spectrum was taken during an integration time of $85 \mathrm{~min}$ and backing pressure of 4 bar; the Ne spectrum was taken during an integration time of $170 \mathrm{~min}$ and backing pressure of 10 bar. Also given in this figure is the transmission curve of the $12.5 \mu \mathrm{m}$ thick beryllium window (black doted line). Taking into account the repetition rate, integration time, and distance of the detector from the target, we estimate the total number of photons/pulse going into $4 \pi$ as 10000 photons from $\mathrm{Kr}$ and 300 photons from Ne. The beryllium absorption is not included in the calculation.

Laser induced x-ray emission from solids and gas targets has a long history, dating back almost to the dawn of lasers, when people started to look at the interaction of nsec and psec lasers with plasmas. In such a laser-matter interaction, the intense laser is heating the plasma into a very high temperature through the inverse bremsstrahlung (IB) or ATI processes. The core-hole excitations are in thermal equilibrium with the surrounding hot plasma. X-ray emission is one of the channels used to keep it in detailed balance. For this model to be valid, it is required that the laser-induced collision rate is much faster than the laser pulse duration, a condition easily met with solid targets and a picosecond long pulse duration. As the pulse duration gets shorter and shorter and the target density gets lower and lower, the conditions reach a certain point after which there is not enough time for heating and thermalization and the resultant plasma temperature drops to the level in which core-hole excitations are not possible anymore. S. Dobosz et al. observed $L$-shell fluorescence from $\mathrm{Kr}$ ions by using $5 \times 10^{17} \mathrm{~W} / \mathrm{cm}^{2}, 130 \mathrm{fs}, \lambda=800 \mathrm{~nm}$ laser, focused on a pulsed gas jet [18]. In electron impact excitation experiments it is known that the characteristic emission may be unisotropic if it results from transitions in which the total angular momentum quantum number of the initial state is $j>1 / 2$. The angular distribution can then be expressed as $I(\theta) / I(\pi / 2)=1-P \cos ^{2} \theta$ where $P$ is the polarization parameter
$[19,20]$. Bremsstrahlung radiation from projectile electrons may also show angular distribution described by the modified Sommerfeld formula [20,21]: $I(\theta) / I(\pi / 2)=$ $\left[(1-\beta \cos \theta)^{2}-P(\cos \theta-\beta)^{2}\right] /\left[\left(1-P \beta^{2}\right)(1-\beta \cos \theta)^{4}\right]$. In the case of excitations due to thermal equilibrium with a hot ambient plasma, we would expect that this angular distribution will average to zero. In their experiments, S. Dobosz et al. and others [22,23] observed an isotropic radiation pattern and therefore attributed this excitation to IB heating of $\mathrm{Kr}$ clusters. C. Prigent et al., investigated the dependence of such excitations on the laser pulse duration [23]. They found a sharp drop in the fluorescence yield as the laser pulses get shorter than $50 \mathrm{fs}$.

The goal of this study is to show that the core-hole excitations we have observed are indeed coming from the recollision process and not from mere heating of the plasma. As a first step towards this goal we followed the reasoning of Ref. [18] and tested the fluorescence and the continuum directionality. For that purpose we used a $\lambda / 2$ wave plate to rotate the polarization direction with respect to the position of the detector and measured both the $\mathrm{x}$-ray fluorescence and the continuum yield as a function of that direction. We observed a minimum in both of them when the polarized field pointed toward the detector and a maximum when it was approximately perpendicular to that direction (see Fig. 3). With the pulse
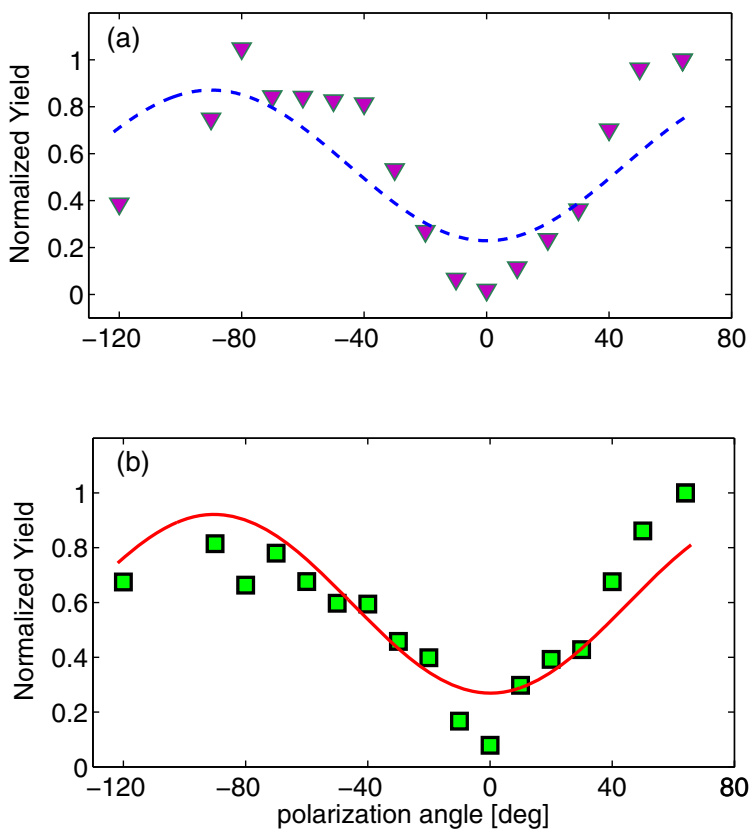

FIG. 3. The krypton $L$-shell fluorescence yield (a) and the continuum yield (b) as a function of the angle between polarization direction and detector direction. We fit the fluorescence yield to the $I(\theta) / I(\pi / 2)=1-P \cos ^{2} \theta$ formula (blue broken line, $P=0.716$ ) and the continuum with the modified Sommerfeld formula which is given above (red solid line, $P=0.78$ ). Measuring conditions: backing pressure of 4 bars, 5 min integration time for each point. 
duration of our IR source (only 2 cycle) and the gas densities we worked at $\left(10^{17}-10^{18} \mathrm{~cm}^{-3}\right)$, we are not expecting IB and ATI heating to play an important role in the observed core-hole excitations. The dipolelike radiation pattern (Fig. 3) strongly supports the recollision excitation mechanism over the IB and ATI heating processes. To further rule out the possibility that the observed $\mathrm{x}$-ray radiation is due to cluster heating, we estimate the cluster size, and accordingly, the role that clusters play in our experiment. Hagena and Obert [24] gave an empirical estimate for the mean cluster size (number of atoms): $\bar{N}=$ $38.4\left(\Gamma^{*} / 1000\right)^{1.64}$ where $\Gamma^{*}=k\left(P_{0} / T_{0}^{2.29}\right)\left(d_{\mathrm{eff}}^{*}\right)^{0.85}$ is the Hagena parameter, $P_{0}$ is the backing pressure [mbar], $T_{0}$ is the pre expansion temperature [kelvin], $k$ is a constant that is gas specific $\left(k_{\mathrm{Kr}}=2890, k_{\mathrm{Ne}}=185\right)$, and $d_{\mathrm{eff}}^{*} \approx$ $[0.74 d / \tan (\alpha)]$ is the effective nozzle diameter [micrometer]. In our experiment we observed $\mathrm{x}$-ray radiation even at a backing pressure of 1 bar (see Supplemental Material [17]), corresponds to $\bar{N}_{\mathrm{Kr}} \approx 540, \bar{N}_{\mathrm{Ne}} \approx 6$. Furthermore we mentioned that, as opposed to Ref. [18], we placed our focused beam just next to the nozzle exit (see Supplemental Material [17]). At such a short distance from the nozzle exit there is not enough time for clusters to condensate and develop, and the above estimation for cluster size should drop by about 2 orders of magnitude [25]. From this estimation, we are not expecting clusters to play an important role in our experiment.

As a next step for testing whether we have recollision excitation or not, we checked how the x-ray yield depends on the drive's ellipticity, since the recollision process is highly sensitive to the polarization ellipticity of the drive [26]. According to the most simplified recollision model, in which, the electron emerges from the deformed Coulomb barrier with a zero velocity, as the ellipticity gets larger and larger, the electron trajectories are pushed away from the parent ion and never come back to recollide with it. Therefore, it is common practice to check whether a process is coming from the recollision process or not by changing the drive polarization ellipticity. Figure 4(a) shows the $\mathrm{x}$-ray yield from the $\mathrm{Kr}$ target as a function of the drive ellipticity.

Indeed, we can see a strong reduction in the x-ray yield as the ellipticity grows. However, as the ellipticity grows, the infrared peak intensity is reduced and leads to a reduced ionization rate which can partially explain the reduction of the photon yield. To check if the reduction in the photon yield is due to the lower ionization yield or due to the deflection of the returning electron, we compare again the results from the linear polarization drive and the circular polarization drive, this time we keep the peak electric field the same [see Fig. 4(b)]. This test shows clearly that the main reduction in the $\mathrm{x}$-ray yield is a result of the electron deflection by the circular polarization. Nevertheless, the signal does not completely disappear with circular polarization as one would expect from the most simplified

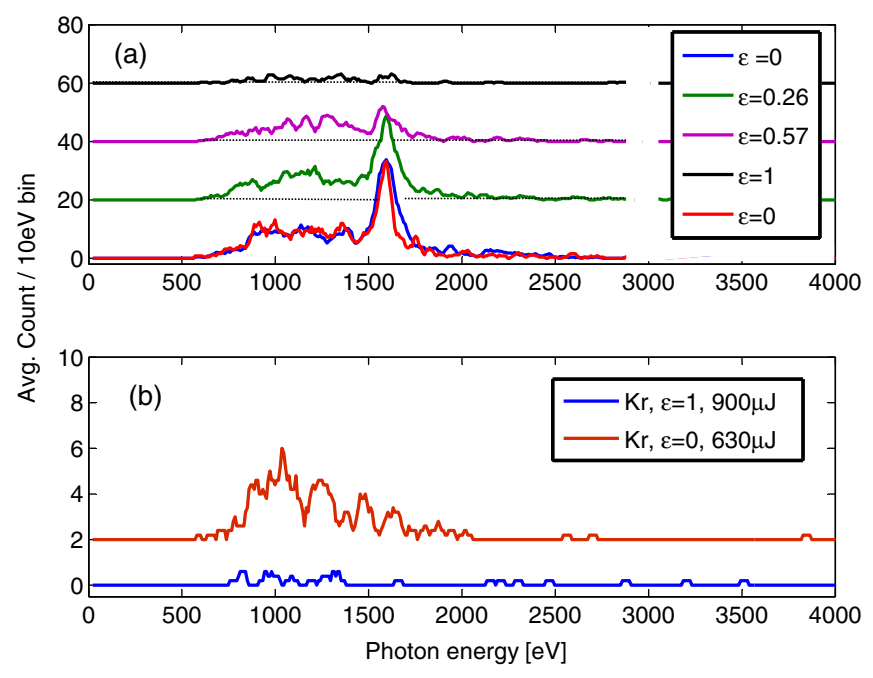

FIG. 4. (a) The $\mathrm{x}$-ray radiation yield from the $\mathrm{Kr}$ atoms vs the drive ellipticity (backing pressure: 4 bar, integration time: $20 \mathrm{~min}$ per spectrum, laser energy $1050 \mu \mathrm{J}$ ). (b) Comparison of the x-ray yield when using a linearly polarized or circularly polarized infrared drive while keeping the peak electric field the same (backing pressure 4 bar, integration time of $60 \mathrm{~min}$ for each spectrum).

recollision induced excitation model. This residual x-ray emission might have a connection to other recently reported findings from other groups. A nonsequential double ionization with circular polarization was reported by a few groups [27-30]. Mizuno et al. observed an extended tail in the spectrum of the photoelectrons from the interaction of a strong circular laser field with $\mathrm{Kr}$ atoms, such a tail was believed to be a signature for electron recollision. An explanation for all these findings might be given by an extended recollision model. In this extended model, the initial velocity of the electron just at the emergence time from the tunneled barrier is not necessarily equal to zero but has some distribution, both along the radiation polarization [31] as well as in the transverse direction [32,33]. Some of these initial velocities allow for recollisions even with circularly polarized radiation [30,34,35]. Another option is a "shake-up" process, in which tunnel ionization results in simultaneous excitation of one or more of the remaining electrons [29]. It is also be possible that the gas jet is thick enough to allow for fast ATI electrons to collide with atoms within the jet and excite them.

In conclusion, the dipolelike radiation pattern (Fig. 3) and the strong dependents of the x-ray emission on the drive ellipticity (Fig. 4) are strong evidence supporting the recollision excitation mechanism over the IB and ATI heating processes. Since the recollision excitation process occurs at the subfemtosecond time scale, it opens the door for time domain studies of electron dynamics in highly excited states where the recollision event initiates the excitation followed by a synchronized attosecond probe pulse. 
We would like to thank Ralph Ernstorfer for critical reading of the Letter and useful insights. G. M. acknowledges support by the Israel Science Foundation (Grant No. 404/12) and the Peter Brojde Center for Innovative Engineering and Computer Science. Z.Z. and R. L. acknowledge support by the National Natural Science Foundation of China (Grants No. 11127901, No. 61221064, and No. 11222439) and National 973 project (No. 2011CB808103).

Y.D., Z.Z., and Z. J. contributed equally to this work.

* Corresponding author.

ruxinli@mail.shcnc.ac.cn

Corresponding author.

gilad.marcus@mail.huji.ac.il

[1] M. Drescher, M. Hentschel, R. Kienberger, M. Uiberacker, V. Yakovlev, A. Scrinzi, T. Westerwalbesloh, U. Kleineberg, U. Heinzmann, and F. Krausz, Nature (London) 419, 803 (2002).

[2] F. Penent, J. Palaudoux, P. Lablanquie, L. Andric, R. Feifel, and J. H. D. Eland, Phys. Rev. Lett. 95, 083002 (2005).

[3] T. Uphues, M. Schultze, M. F. Kling, M. Uiberacker, S. Hendel, U. Heinzmann, N. M. Kabachnik, and M. Drescher, New J. Phys. 10, 025009 (2008).

[4] A. J. Verhoef, A. V. Mitrofanov, X. T. Nguyen, M. Krikunova, S. Fritzsche, N. M. Kabachnik, M. Drescher, and A. Baltuška, New J. Phys. 13, 113003 (2011).

[5] F. Krausz and M. Ivanov, Rev. Mod. Phys. 81, 163 (2009).

[6] G. Marcus, W. Helml, X. Gu, Y. Deng, R. Hartmann, T. Kobayashi, L. Strueder, R. Kienberger, and F. Krausz, Phys. Rev. Lett. 108, 023201 (2012).

[7] A. D. Shiner, B. E. Schmidt, C. Trallero-Herrero, H. J. Worner, S. Patchkovskii, P. B. Corkum, J.-C. Kieffer, F. Legare, and D. M. Villeneuve, Nat. Phys. 7, 464 (2011).

[8] J. L. Krause, K. J. Schafer, and K. C. Kulander, Phys. Rev. Lett. 68, 3535 (1992).

[9] P. B. Corkum, Phys. Rev. Lett. 71, 1994 (1993).

[10] J. H. Eberly, J. Javanainen, and K. Rzazewski, Phys. Rep. 204, 331 (1991).

[11] A. L'Huillier, L. A. Lompre, G. Mainfray, and C. Manus, Phys. Rev. A 27, 2503 (1983).

[12] D. N. Fittinghoff, P. R. Bolton, B. Chang, and K. C. Kulander, Phys. Rev. Lett. 69, 2642 (1992).

[13] M. F. Kling et al., Science 312, 246 (2006).
[14] J. Weisshaupt, V. Juvé, M. Holtz, S. Ku, M. Woerner, T. Elsaesser, S. Ališauskas, A. Pugžlys, and A. Baltuška, Nat. Photonics 8, 927 (2014).

[15] C. Zhang, P. Wei, Y. Huang, Y. Leng, Y. Zheng, Z. Zeng, R. Li, and Z. Xu, Opt. Lett. 34, 2730 (2009).

[16] E. Gatti and P. Rehak, Nucl. Instrum. Methods Phys. Res., Sect. A 541, 47 (2005).

[17] See Supplemental Material at http://link.aps.org/ supplemental/10.1103/PhysRevLett.116.073901 for raw spectra; spectra obtained at low pressure; and nozzle-beam relative positioning.

[18] S. Dobosz, M. Lezius, M. Schmidt, P. Meynadier, M. Perdrix, D. Normand, J.-P. Rozet, and D. Vernhet, Phys. Rev. A 56, R2526 (1997).

[19] W. Mehlhorn, Phys. Lett. A 26, 166 (1968).

[20] Coherence and Correlation in Atomic Collisions, edited by H. Kleinpoppen and J. F. Williams (Springer US, Boston, MA, 1980), ISBN , .

[21] H. Kulenkampff, M. Scheer, and E. Zeitler, Z. Phys. 157, 275 (1959).

[22] E. Springate, S. A. Aseyev, S. Zamith, and M. J. J. Vrakking, Phys. Rev. A 68, 053201 (2003).

[23] C. Prigent, C. Deiss, E. Lamour, J.-P. Rozet, D. Vernhet, and J. Burgdörfer, Phys. Rev. A 78, 053201 (2008).

[24] O. F. Hagena and W. Obert, J. Chem. Phys. 56, 1793 (1972).

[25] A. Ramos, J. M. Fernández, G. Tejeda, and S. Montero, Phys. Rev. A 72, 053204 (2005).

[26] K. S. Budil, P. Salières, M. D. Perry, and A. L'Huillier, Phys. Rev. A 48, R3437 (1993).

[27] G. D. Gillen, M. A. Walker, and L. D. Van Woerkom, Phys. Rev. A 64, 043413 (2001).

[28] C. Guo and G. N. Gibson, Phys. Rev. A 63, 040701 (2001).

[29] W. A. Bryan et al., Nat. Phys. 2, 379 (2006).

[30] F. Mauger, C. Chandre, and T. Uzer, Phys. Rev. Lett. 105, 083002 (2010).

[31] A. N. Pfeiffer, C. Cirelli, A. S. Landsman, M. Smolarski, D. Dimitrovski, L. B. Madsen, and U. Keller, Phys. Rev. Lett. 109, 083002 (2012).

[32] M. Y. Ivanov, M. Spanner, and O. Smirnova, J. Mod. Opt. 52, 165 (2005)

[33] L. Arissian, C. Smeenk, F. Turner, C. Trallero, A. V. Sokolov, D. M. Villeneuve, A. Staudte, and P. B. Corkum, Phys. Rev. Lett. 105, 133002 (2010).

[34] N. I. Shvetsov-Shilovski, S. P. Goreslavski, S. V. Popruzhenko, and W1. Becker, Phys. Rev. A 77, 063405 (2008).

[35] X. Wang and J. H. Eberly, New J. Phys. 12, 093047 (2010). 